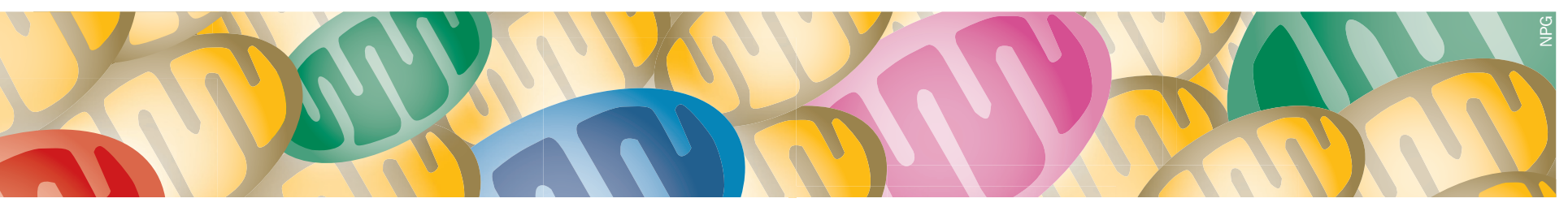

\title{
Defective mitochondrial dynamics in the hot seat-a therapeutic target common to many neurological disorders?
}

T wo new studies of neurological diseases characterized by mitochondrial dysfunction have revealed therapeutic approaches to restore function of these organelles, and provide hope for treatments for other neurological disorders that are associated with aberrant mitochondrial dynamics.

"Mitochondrial damage has been observed in numerous neurological disorders including Huntington disease and Parkinson disease (PD); however, whether selective correction of mitochondrial dysfunction protects against neuronal damage is poorly demonstrated," says Xin Qi, senior author of one of the studies.

Qi's group at Case Western Reserve University School of Medicine, OH, USA, has recently developed a selective peptide inhibitor of excessive mitochondrial fission, termed P110. This inhibitor targets Drp1-a primary mitochondrial fission-related protein that, when recruited to mitochondria, leads to mitochondrial fragmentation.

Previously, Qi and colleagues showed that P110 treatment inhibits mitochondrial and neuronal damage in in vitro models of neurotoxin-induced PD. In the current study, they asked whether inhibition of Drp1-mediated mitochondrial fission is protective in PD caused by a Gly2019Ser mutation in leucine-rich repeat kinase 2 (LRRK2), which accounts for $2 \%$ of sporadic PD.

Using LRRK2 Gly2019Ser-transfected cell lines, Qi and colleagues showed that hyperactivation of Drp1 mediates the excessive mitochondria-associated autophagy that is evident in patients with this mutation. The Gly2019Ser mutant was found to activate Drp1 by phosphorylation at Thr595, providing the first evidence that Drp1 can be phosphorylated at this site.

Next, the researchers differentiated induced pluripotent stem cells, derived from patients with the LRRK2 mutation, into dopaminergic neurons. Treatment of these cells with P110 reduced the number of mitochondria along the neurons and substantially reduced mitochondrial fragmentation.

The group are testing the efficacy of P110 in animal models of PD, but Qi is optimistic that this drug has implications beyond a treatment for PD. "Aberrant mitochondrial dynamics are associated with a number of neurological disorders, and we are studying if P110 treatment is neuroprotective in other diseases in which Drp1-mediated mitochondrial dysfunction occurs," she says.

In the other study, led by Rita Horvath who is based at the Institute of Genetic Medicine, Newcastle University, UK, the researchers investigated the effect of a novel mitochondria-targeted therapy in primary mitochondrial disorders. Childhood-onset mitochondrial encephalomyopathies are severe and relentlessly progressive conditions, for which treatments are lacking. Horvath noted, however, that some types of severe mitochondrial disease can spontaneously reverse. Can study of these diseases inform therapeutic approaches to nonreversible mitochondrial disease?

"In two types of reversible infantile mitochondrial disease (namely, reversible infantile respiratory chain deficiency [RIRCD] and reversible infantile hepatopathy) there is a common factor: involvement of mitochondrial transfer
$\mathrm{RNA}^{\mathrm{Glu}}$ (mt-tRNA ${ }^{\mathrm{Glu}}$ )," explains Horvath. "Given the similar infantile clinical presentation and reversibility of these two conditions, we decided to study whether a specific modification of this tRNAnamely, 2-thiouridylation-contributes to disease manifestation."

Using a high-resolution Northern blotting technique, the researchers showed that downregulation of TRMU (a molecule required for 2-thiouridylation) in fibroblasts and myoblasts from patients with RIRCD led to a reduction in levels of mt-tRNA ${ }^{\text {Glu }}$ thiolation, resulting in a defect of mitochondrial protein synthesis.

Cysteine is needed for thiolation, and the investigators next showed that L-cysteine supplementation improved mitochondrial gene translation in cells from patients with RIRCD.

"Our data suggest that L-cysteine has a beneficial effect on mitochondrial translation in reversible mitochondrial myopathy and maybe also in other forms of mitochondrial disease," says Horvath, "We are planning further experiments to study the effect of L-cysteine in different mitochondrial diseases."

If successful, future studies from these two groups could present novel therapeutics for mitochondrial or mitochondria-associated disorders, many of which are currently incurable.

Katy Malpass

Original articles Boczonadi, V. et al. Altered 2-thiouridylation
impairs mitochondrial translation in reversible infantile
respiratory chain deficiency. Hum. Mol. Genet. doi:10.1093/
hmg/ddt309 | Su,Y.-C. \& Qi, X. Inhibition of excessive
mitochondrial fission reduced aberrant autophagy and
neuronal damage caused by LRRK2 G2019S mutation.
Hum. Mol. Genet. doi:10.1093/hmg/ddt301

